\title{
Association of Physical activity levels and BMI Among Doctor of Physical Therapy Students of a Private College from Karachi
}

\author{
Khalid Aziz, Ayesha Afridi, Abida Arif, Ghousia Shahid, Muhammad Faisal Fahim, Rabia Khan
}

ABSTRACT:

Objective: To evaluate the physical activity levels among DPT students of Bahria University College of Physical therapy (BUCPT) by International Physical Activity Questionnaire (IPAQ) and to study the relationship between Body Mass Index (BMI) and Physical activity levels of DPT students of (BUCPT).

Study design and Setting: This cross sectional study was conducted in BUCPT Bahria from March- April 2019 (1 month data collection).

Methodology: Written consent form was obtained from each participant before taking part into study. Individual with cardio-respiratory diseases and fractures in last 12 months or any limb disability were excluded from the study. The study tool was IPAQ.

Results:A total of 125 students were enrolled in this study. There were $121(96.8 \%)$ female and $4(3.2 \%)$ male observed. Mean age of students were $19.89 \pm 1.14(18-22)$ years. In low physical activity level, $2(28.6 \%)$ students were underweight, $8(17.0 \%)$ were normal BMI, $3(9.7 \%)$ were overweight and $6(15.0 \%)$ were obese. In moderate physical activity level, 3 (42.9\%) underweight, 22 (46.8\%) normal, 19 (61.3\%) overweight and 19 (47.5\%) fall in obese criteria. High physical activity levels were found to be $2(28.6 \%)$ in underweight group, 17 (36.2\%) in normal group, 9 (29.0\%) in overweight and $15(37.5 \%)$ were obese.

Conclusion: BUCPT students were moderately physically active and minority of them were low physically active and majority of them were lying the category of normal BMI. There was statistically insignificant relationship found between BMI and physical activities levels. Some students were obese and still have high physical activity level while some are normal in BMI and having low physical activity level.

Key words: Assessment, BMI, Physical Activity, IPAQ questionnaire, Exercises, Obesity, DPT

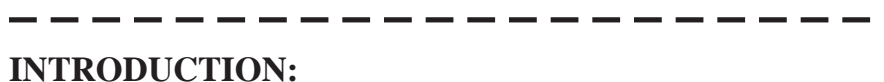

\section{INTRODUCTION:}

Proper workout of skeletal muscles to produce productive bodily movements by utilizing calories and results in energy expenditure is termed as physical activity or sometimes interchangeably called exercise ${ }^{1}$. Opposite to that, physical inactivity results in adverse health conditions like diabetes mellitus, breast cancer, obesity, hypertension and hyperchol-

Khalid Aziz

I Vice Principal,

I Bahria University College of Physical Therapy

Email: vp.bucpt@bumdc.edu.pk

Ayesha Afridi

Lecturer

I Bahria University College of Physical Therapy

I Abida Arif

I Senior Lecturer

I Bahria University College of Physical Therapy

I Ghousia Shahid

Senior Lecturer,

Bahria University College of Physical Therapy

Muhammad Faisal Fahim

Researcher,

I Bahria University College of Physical Therapy

I Rabia Khan

I Lecturer,

I Bahria University College of Physical Therapy

I Received: 27-05-2019

Accepted: 01-10-2019

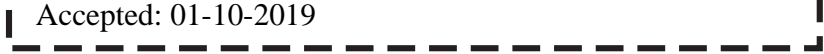

esterolemia which ultimately leads to coronary heart disease ${ }^{2}$. The puberty is the transition link for individuals to move from childhood to adulthood and majority of the health habits developed at this transition level, lasts long e.g. exercise and dietary habits ${ }^{3}$. Many previous researches indicate that today's lifestyle of adults is deprived of health consciousness and total dependency upon technology is diminishing the physical activity levels ${ }^{4}$. Exercise and physical activities are practically ignored in daily routine of adult university students due to different corresponding factors including lack of supervision for proper exercise plan, poor time management and disbelieve about positive effects of physical activity upon body ${ }^{5}$. Overweight and obesity among young adults' leads to low self-esteem and it directly affects the confidence level and academic record of university students. Physical activity/ exertion boosts the metabolic system for young students in their growing age and maintain the hormone balance in female students ${ }^{6}$. The transition from childhood to adolescences decreases the level of physical activities and with further advancing age physical activity level further deteriorated Growing age along with biological changes and psychological maturation and habits developmental stage, the physical activity levels decreases and leading to unhealthy, sedentary life style in university students ${ }^{7}$. Different perceived barriers to physical activity among students are classified as internal and external 
barriers. Daskapan at al reported in their study that score for external barriers like lack of time for exercise was significantly higher than internal barrier which include lack of stamina and energy for exercise ${ }^{3}$. Health professionals are making efforts to aware the population about the hazardous effects of physical inactivity and sedentary life style by counseling the individuals visiting them for health issues in last decades ${ }^{8}$. Unfortunately the facilities provided by latest technologies are overcoming the manual work outs of adults and physical activity attitude and behaviors are difficult to adopt and inculcate in daily routine ${ }^{9}$. World health organization has provided the guidelines for adults of age 18 to 64 years for healthy life style. These guidelines recommend that adults should do moderate intensity physical activity for at least 150 minutes and vigorous intensity physical exertion for 75 minutes per week or mixture of both intensities at regular level ${ }^{10}$. Previous research reported that high academic stress among university students discourages the will to do physical exertion and exercise, which leads to weight gain and ultimately further decline in the stamina for physical activities ${ }^{11}$. Worldwide the prevalence of physical inactivity leading to obesity is increasing drastically among university students ${ }^{12}$. The prevalence for obesity among university students from 22 different countries has been reported through selfadministered questionnaires and Body Mass Index (BMI) for weight status. Overall in male and female, $22 \%$ students were lying in category of overweight or obesity due to physical in activity ${ }^{13}$. Studies in low, middle and high income countries showed drastic results of physical inactive university students, Pakistani students are counted as $80.6 \%$ physically inactive due to regularly skipping breakfast and lack of social support for personal and academic workload ${ }^{14}$. Previous studies reported that high prevalence of physical inactivity and obesity is linked with poor diet control routine, sleep deprivation and academic stress among university students ${ }^{15}$. University students are having more freedom for their dietary and sleeping time choices ${ }^{16,17}$. According to WHO 2010 guidelines for physical activities in adults which include at least 150 minutes of moderate and minimum 75 minutes of vigorous intensity activities in one week for healthy life style and good quality of life ${ }^{18}$. Inadequate exercise or physical activity routine are causing mortality rates even higher each year. In 2010 almost 3.2 million deaths were reported by WHO solely due to inadequate physical activity. Physical inactivity is leading to vicious cycle of cardio respiratory, obesity and hypercholesterolemia issues in young adults which can affect their personal, social and academic life adversely ${ }^{19,20}$. Maintaining the regular physical activity level can prevent from many chronic diseases and quality of life can also be improved. Quality of life is the perception of individuals towards their positive and negative aspects of life ${ }^{2}$. International Physical Activity Questionnaire (IPAQ) is validated tool used in many researchers for methodological measurement of physical activity levels among adults ${ }^{21}$ Being a physical therapy student everyone should know the importance of exercise and physical activity for healthy and good quality of life because in future they have to deal with patients of same issues. The rationale of the study was to create awareness about exercise, physical activity and obesity in BUCPT students by using validated IPAQ tool. This study was aimed to evaluate the physical activity levels among DPT students of Bahria University College of Physical therapy (BUCPT) by international physical activity questionnaire (IPAQ) and to study the relationship between Body Mass Index (BMI) and Physical activity levels of DPT students of (BUCPT).

\section{METHODOLOGY:}

Ethical approval was obtained from BUMDC numbered: 11/2019. This cross sectional study was conducted in Bahria university college of Physical Therapy (BUCPT) from March- April 2019 (1 month data collection). Written consent form was signed by each participant before taking part into study. Total 125 Doctor of Physical Therapy (DPT) students were enrolled in study by purposive sampling technique with mean age of 19.9 \pm 1.4 (18-22 years). Inclusion criteria was individuals should be above age 15 (requirement of international Physical Activity questionnaire IPAQ), from (BUCPT) and willing to be the part of this study. Individual with Cardio-respiratory diseases, fractures in last 12 months and any limb disability were excluded from study. After signing the consent form, participants were assessed for anthropometric measurement such as weight in $\mathrm{kg}$ and height in meters by Physical Therapist of BUCPT. Body Mass Index was determined using formula weight $(\mathrm{kg}) / \mathrm{Height}(\mathrm{m})$. Physical activity of students was assessed using IPAQ International Physical activity questionnaire short version comprising of total 7 questions. This questionnaire has 3 categories of activities, including vigorous, moderate and walking and 4th one was related to sitting hours in weekdays. Through this questionnaire MET minutes were calculated for each activity and then individuals were assigned either low physical active, moderate or high physical active according to MET minutes calculation ${ }^{22}$. One metabolic equivalent (MET) is defined as the amount of oxygen consumed while sitting at rest and is equal to $3.5 \mathrm{ml} \mathrm{O}_{2}$ per $\mathrm{kg}$ body weight per min. The MET concept represents a simple, practical, and easily understood procedure for expressing the energy cost of physical activities as a multiple of the resting metabolic rate. Individual categorical calculations were done using automatic scoring sheet of excel for International Physical activity Questionnaire designed by Cheng $\mathrm{H}^{23}$ Scoring was taken and calculated in MET-minutes/week (www.ipaq.ki.se). The subsequent value were used for the investigation of IPAQ data:

- Walking MET $=3.3 \mathrm{x}$ walking minutes $\mathrm{x}$ walking days

- Moderate MET $=4.0 \mathrm{x}$ walking minutes $\mathrm{x}$ walking days 
Khalid Aziz, Ayesha Afridi, Abida Arif, Ghousia Shahid, Muhammad Faisal Fahim, Rabia Khan

- Vigorous MET $=8.0 \mathrm{x}$ walking minutes $\mathrm{x}$ walking days

- $\quad$ Total Physical Activity MET = sum of Walking + Moderate + Vigorous MET minutes/week scores ${ }^{24}$.

Statistical analysis was performed on SPSS-23. All variables of International Physical activity questionnaire were evaluated according to their classification. Frequency and percentages and Mean and Standard deviation are presented in results.

\section{RESULTS:}

A total of 125 students were enrolled in this study. There were $121(96.8 \%)$ female and $4(3.2 \%)$ male observed. Mean age of students were $19.89 \pm 1.14(18-22)$ years. Students mean height was found to be $1.48 \pm 0.10$ (1.27-1.89) meters. Mean Weight of students was found to be 53.73 \pm 10.31 (30 - 80) $\mathrm{kg}$. Average BMI in students was found to be $24.65 \pm 4.85$. Physical activity MET according to IPAQ was asked from students that how many days did you do vigorous physical activities like heavy lifting, digging, aerobics, or fast bicycling in last 7 days mean value found to be $2.37 \pm 1.48$ days per week. When asking about how much time did you usually spend doing vigorous physical activities on one of those days mean value was found to be $10.37 \pm 3.43$ minutes per day. When asking about how many days did you do moderate physical activities like carrying light loads, bicycling at a regular pace, or doubles tennis? Mean value of $3.22 \pm 1.53$ days per week observed. Students were asked that how much time did you usually spend doing moderate physical activities on one of those days. Average value was found to be 23.15 \pm 8.36 minutes per week. They were asked that how many days you walked for at least 10 minutes at a time. Mean value was found to be $5.70 \pm 1.82$ days per week. The question asked was how much time you usually spent walking on one of those days. Average value was observed to be $50.21 \pm 5.10$ minutes per day. Students were asked about how much time you spent sitting on a week day. Mean value was found to be $15.49 \pm 3.74$ minutes per day. Mean total days of activity was $6.63 \pm 1.05$. Mean Met-minutes per week vigorous was $704.35 \pm 112.36$. Mean Total activity was $83.74 \pm 17.36$ ( $\mathrm{min} /$ week). Mean Met-minutes per week was 408.48 \pm 78.07 . Mean Met-minutes per week walk was 1019.01 \pm 106.36 . Mean Met-minutes per week moderate total was $1806.82 \pm 152.40$. (Table 1)

Body Mass Index (BMI) was followed by Asian cut off values as Underweight, Normal, over weight and obese. There were $47(37.6 \%)$ students lying in the normal category whereas $40(32.0 \%)$ found to be obese. Physical activity levels were observed as low $19(15 \%)$, moderate $63(51 \%)$ and high 43 (34\%) students. (Figure 1)

There was statistically insignificant relationship found between BMI and physical activities levels. Some students are obese and still have high physical activity level while some are normal in BMI and having low physical activity level. (Table 2)

\section{DISCUSSION:}

Current study aim was to identify the physical activity levels in DPT students using IPAQ; any activity that lasts for more than 10 minutes was included. Results showed that $34 \%$ students were involved in vigorous physical activity on further elaborating the activities, majority of the students showed more than 100 stairs climbing activity in 1 hour due to construction design of BUMDC (nine floors), 51\% with moderate physical activity level and only $15 \%$ were found with low physical activity level. On contrary study by Supa

Table 1: Interpretation of Total Physical activity MET according with IPAQ

\begin{tabular}{|l|c|}
\hline $\begin{array}{l}\text { Question regarding vigorous activity of last } 7 \text { days / Interpretation of Total Physical activity } \\
\text { MET according with IPAQ }\end{array}$ & Mean \pm SD \\
\hline $\begin{array}{l}\text { During the last 7 days, on how many days did you do vigorous physical activities like heavy lifting, } \\
\text { digging, aerobics, or fast bicycling? }\end{array}$ & $2.37 \pm 1.48$ \\
\hline How much time did you usually spend doing vigorous physical activities on one of those days? & $10.37 \pm 3.43$ \\
\hline $\begin{array}{l}\text { During the last 7 days, on how many days did you do moderate physical activities like carrying } \\
\text { light loads, bicycling at a regular pace, or doubles tennis? Do not include walking. }\end{array}$ & $3.22 \pm 1.53$ \\
\hline How much time did you usually spend doing moderate physical activities on one of those days? & $23.15 \pm 8.36$ \\
\hline During the last 7 days, on how many days did you walk for at least 10 minutes at a time? & $5.70 \pm 1.82$ \\
\hline How much time did you usually spend walking on one of those days? & $50.21 \pm 5.10$ \\
\hline During the last 7 days, how much time did you spend sitting on a week day? & $15.49 \pm 3.74$ \\
\hline Total days of activity & $6.63 \pm 1.05$ \\
\hline Met-minutes per week vigorous & $704.35 \pm 112.36$ \\
\hline Total activity (min/week) & $83.74 \pm 17.36$ \\
\hline Total Met-minutes per week & $408.48 \pm 78.07$ \\
\hline Met-minutes per week walk & $1019.01 \pm 106.36$ \\
\hline Met-minutes per week moderate total & $1806.82 \pm 152.40$ \\
\hline
\end{tabular}


Table 2: BMI in comparison with Physical activity levels.

\begin{tabular}{|c|c|c|c|c|c|}
\hline & \multicolumn{3}{|c|}{ PHYSICAL ACTIVITY LEVELS } & \multirow[b]{2}{*}{ Total $(n=125)$} & \multirow[b]{2}{*}{ P-value } \\
\hline BMI (kg/m2) & Low $(n=19)$ & Moderate $(n=63)$ & High $(n=43)$ & & \\
\hline \multirow{2}{*}{$\begin{array}{c}\text { Under weight } \\
(<18.5)\end{array}$} & 2 & 3 & 2 & 7 & \multirow{8}{*}{0.575} \\
\hline & $28.6 \%$ & $42.9 \%$ & $28.6 \%$ & $100.0 \%$ & \\
\hline \multirow{2}{*}{$\begin{array}{c}\text { Normal } \\
(18.5-22.9)\end{array}$} & 8 & 22 & 17 & 47 & \\
\hline & $17.0 \%$ & $46.8 \%$ & $36.2 \%$ & $100.0 \%$ & \\
\hline \multirow{2}{*}{$\begin{array}{c}\text { Over Weight } \\
(23-26.9)\end{array}$} & 3 & 19 & 9 & 31 & \\
\hline & $9.7 \%$ & $61.3 \%$ & $29.0 \%$ & $100.0 \%$ & \\
\hline \multirow{2}{*}{$\begin{array}{l}\text { Obese } \\
(=27)\end{array}$} & 6 & 19 & 15 & 40 & \\
\hline & $15.0 \%$ & $47.5 \%$ & $37.5 \%$ & $100.0 \%$ & \\
\hline
\end{tabular}

Figure 1: Physical Activity Levels Of Students

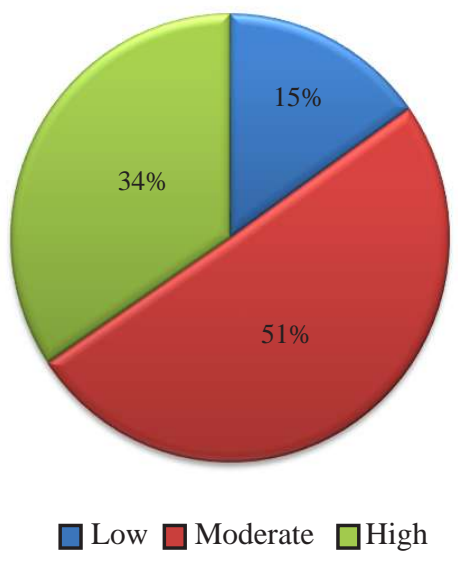

et al (2015) revealed that physical activity levels among university students using IPAQ, total 813 students were enrolled from Pakistan with $80.6 \%$ low, $7.7 \%$ moderate and $11.7 \%$ with high physical activity levels ${ }^{14}$. another study was done in 2016 on Turkish student's physical activity levels using IPAQ and results were near to current study results; $52 \%$ students were found to be moderately physical active $37 \%$ vigorous and only $11 \%$ with low physical activity level $^{25}$. Ajit et al conducted a study in 2018 on Indian Physical Therapy students using IPAQ, results showed that $49 \%$ were moderately physically active , $39 \%$ low physically active and $12 \%$ with high physical activity level ${ }^{26}$.

Current study showed the BMI of students which was alarming as $5.6 \%$ students were under weight, $37.6 \%$ were normal, $24.8 \%$ were overweight and $32 \%$ were lying in the category of obese. There was statistically insignificant relationship found between BMI physical activity level. Some students are obese and still have high physical activity level while some are normal in BMI and having low physical activity level. Indian study on physical therapy students showed that $64 \%$ were normal, $9 \%$ were obese, $14 \%$ were overweight and $13 \%$ were underweight according to BMI calculations. Out of 289 normal subjects, 39\% were low,
$51 \%$ moderate and $10 \%$ were highly physically active. Out of 40 obese subjects $32 \%$ were low, $42 \%$ moderate, and $26 \%$ were high physically active. Out of 59 underweight subjects, $53 \%$ were low, $39 \%$ moderate, and $8 \%$ were high physically active. Out of 62 overweight subjects, $27 \%$ were low, $56 \%$ moderate, and $17 \%$ were high physically active ${ }^{26}$.

The validated questionnaire of IPAQ was the strength of the study and smaller sample size, single centered study were the limitations of the study. It was recommended the physical activity is important for students of all subjects and for this in house gymnasium should be structured for the students. Multiple centered study and students of various students program should be carried out in future for generalizability of the results.

\section{CONCLUSION:}

Majority of the Doctor of Physical Therapy (DPT) students were moderately physicaly active and minority of them were low physicaly active and majority of them were lying in the category of normal BMI. There was statistically insignificant relationship found between BMI and physical activities levels. Some students were obese and still have high physical activity level while some are normal in BMI and having low physical activity level.

\section{REFERENCES:}

1. Caspersen, C.J., K.E. Powell, and G.M. Christenson, Physical activity, exercise, and physical fitness: definitions and distinctions for health-related research. Public health reports, 1985; 100(2): p. 126.

2. Joseph, R.P., et al., Physical activity and quality of life among university students: exploring self-efficacy, self-esteem, and affect as potential mediators. Quality of life research, 2014; 23(2): p. 659-667.

3. Arzu, D., E.H. Tuzun, and L. Eker, Perceived barriers to physical activity in university students. Journal of sports science \& medicine, 2006; 5(4): p. 615.

4. Trost, S.G., et al., Age and gender differences in objectively measured physical activity in youth. Medicine \& Science in Sports \& Exercise, 2002; 34(2): p. 350-355.

5. Dishman, R.K., Advances in exercise adherence. 1994: human kinetics publishers. 
6. Van Dyck, D., et al., International study of objectively measured physical activity and sedentary time with body mass index and obesity: IPEN adult study. International Journal of Obesity, 2015; 39(2): p. 199.

7. Arias-Palencia, N.M., et al., Levels and patterns of objectively assessed physical activity and compliance with different public health guidelines in university students. PLoS ONE, 2015; 10(11): p. e0141977.

8. Keating, X.D., et al., A meta-analysis of college students' physical activity behaviors. Journal of American college health, 2005; 54(2): p. 116-126.

9. Daley, A.J., School based physical activity in the United Kingdom: Can it create physically active adults? Quest, 2002; 54(1): p. 21-33.

10. Organization, W.H., Global recommendations on physical activity for health. 2010.

11. Romaguera, D., et al., Determinants and patterns of physical activity practice among Spanish university students. Journal of sports sciences, 2011; 29(9): p. 989-997.

12. Haidar, Y.M. and B.C. Cosman, Obesity epidemiology. Clinics in colon and rectal surgery, 2011; 24(04): p. 205-210.

13. Peltzer, K., et al., Prevalence of overweight/obesity and its associated factors among university students from 22 countries. International journal of environmental research and public health, 2014; 11(7): p. 7425-7441.

14. Pengpid, S., et al., Physical inactivity and associated factors among university students in 23 low-, middle-and high-income countries. International journal of public health, 2015; 60(5): p. 539-549.

15. Kattelmann, K.K., et al., Development of Young Adults Eating and Active for Health (YEAH) internet-based intervention via a community-based participatory research model. Journal of nutrition education and behavior, 2014; 46(2): p. S10-S25.

16. Cavallo, D.N., et al., A social media-based physical activity intervention: a randomized controlled trial. American journal of preventive medicine, 2012; 43(5): p. 527-532.
17. Khan, Z.N., et al., High prevalence of preobesity and obesity among medical students of Lahore and its relation with dietary habits and physical activity. Indian journal of endocrinology and metabolism, 2016; 20(2): p. 206.

18. Organization, W.H., Global recommendations on physical activity for health. 2010: World Health Organization.

19. Wattanapisit, A., et al., Physical activity among medical students in Southern Thailand: a mixed methods study. BMJ Open, 2016; 6(9): p. e013479.

20. Bergier, J., et al., Physical activity of Polish adolescents and young adults according to IPAQ: a population based study. Annals of Agricultural and Environmental Medicine, 2012; 19(1):109-115

21. Bergier, J., B. Bergier, and T. Anatolii, Variations in physical activity of male and female students from different countries. Iranian journal of public health, 2016; 45(5): p. 705-707.

22. Murphy, J.J., et al., Validity and reliability of three self-report instruments for assessing attainment of physical activity guidelines in university students. Measurement in Physical Education and Exercise Science, 2017; 21(3): p. 134-141.

23. Cheng, H., A simple, easy-to-use spreadsheet for automatic scoring of the International Physical Activity Questionnaire (IPAQ) Short Form (updated November 2016). 2016, ResearchGate.

24. Fagaras, S.-P., L.-E. Radu, and G. Vanvu, The level of physical activity of university students. Procedia-Social and Behavioral Sciences, 2015; 197: p. 1454-1457.

25. Bednarek, J., et al., Physical activity of Polish and Turkish university students as assessed by IPAQ. Central European Journal of Sport Sciences and Medicine, 2016; 16(4): p. 13 22.

26. Dabholkar, A., N.N. Valanju, and T. Dabholkar, Assessment of Level of Physical Activity in Physiotherapy Students. 2018, 2018; 5(1): p. 4. 